\title{
Concepções de docentes sobre o processo ensino aprendizagem em cenários de prática da graduação em nutrição
}

\author{
Teacher's conceptions about the teaching-learning process in undergraduate nursing \\ practice scenarios
}
Concepciones del profesor sobre el proceso de enseñanza-aprendizaje en escenarios de práctica de enfermería de pregrado

Michele de Freitas Melo1, Luiz Euclides Coelho de Souza Filho', Robson José de Souza Domingues $^{1}$, Valéria Marques Ferreira Normando ${ }^{1 *}$.

\begin{abstract}
RESUMO
Objetivo: Analisar as concepções dos docentes na formação do profissional nutricionista a cerca do processo ensino aprendizagem, durante o estágio supervisionado em Nutrição Social. Métodos: A pesquisa exploratória com abordagem qualitativa, desenvolvida com vinte e um docentes nutricionistas da Faculdade de Nutrição de uma universidade pública do estado do Pará respondeu a um questionário semiestruturado com o auxílio do software ALCESTE. Resultados: Cinco classes de segmentos de texto com contextos semânticos específicos foram alcançados. Destacou-se a classe 1 como a mais específica quanto a importância do estágio como uma atividade de consolidação do conhecimento adquirido no transcorrer da formação acadêmica. Ao articular a universidade aos diversos cenários de prática, verificou-se impactos na formação e qualificação profissional. Percebeu-se que as universidades devem estreitar os vínculos com os serviços de saúde, para melhor capacitação profissional. Novas tendências pedagógicas espelham metodologias que possibilitem o empoderamento do aluno, colocando-o como protagonista central da construção de seu conhecimento e corresponsável pela sua trajetória educacional e o professor, um mediador das experiências relacionadas ao processo de aprendizagem. Conclusão: Planejar e discutir estratégias acadêmicas no processo ensino-aprendizagem são motivadas pelas ações de formação continuada ao docente.
\end{abstract}

Palavras-chave: Ensino, Educação superior, Docentes, Nutricionista.

\begin{abstract}
Objective: To analyze the teachers' conceptions in the training of the nutritionist about the teaching-learning process, during the supervised internship in Social Nutrition. Methods: The exploratory research with a qualitative approach, developed with twenty-one nutritionist professors from the Faculty of Nutrition of a public university in the state of Pará, answered a semi-structured questionnaire with the aid of the ALCESTE software. Results: Five classes of text segments with specific semantic contexts were achieved. Class 1 was highlighted as the most specific in terms of the importance of the internship as an activity to consolidate the knowledge acquired in the course of academic training. When articulating the university with the different practice scenarios, there were impacts on professional training and qualification. It was noticed that universities should strengthen their links with health services, for better professional training. New pedagogical trends mirror methodologies that enable the empowerment of the student, placing him as the central protagonist of the construction of his knowledge and co-responsible for his educational trajectory and the teacher, a mediator of the experiences related to the learning process. Conclusion: Planning and
\end{abstract}

1 Universidade do Estado do Pará (UEPA), Belém - PA. *E-mail: valerianormando@uepa.br 
discussing academic strategies in the teaching-learning process are motivated by continuing education actions for teachers.

Keywords: Teaching, College education, Teachers, Nutritionist.

\section{RESUMEN}

Objetivo: Analizar las concepciones de los docentes en la formación del nutricionista sobre el proceso de enseñanza-aprendizaje, durante la pasantía supervisada en Nutrición Social. Métodos: La investigación exploratoria con un enfoque cualitativo, desarrollada con veintiún profesores nutricionistas de la Facultad de Nutrición de una universidad pública en el estado de Pará, respondió un cuestionario semiestructurado con la ayuda del software ALCESTE. Resultado: Se lograron cinco clases de segmentos de texto con contextos semánticos específicos. La Clase 1 se destacó como la más específica en términos de la importancia de la pasantía como actividad para consolidar los conocimientos adquiridos en el curso de la formación académica. Al articular la universidad con los diferentes escenarios de práctica, hubo impactos en la capacitación y calificación profesional. Se dio cuenta de que las universidades deberían fortalecer sus vínculos con los servicios de salud, para una mejor capacitación profesional. Las nuevas tendencias pedagógicas reflejan metodologías que permiten el empoderamiento del alumno, ubicándolo como el protagonista central de la construcción de su conocimiento y corresponsable de su trayectoria educativa y el maestro, un mediador de las experiencias relacionadas con el proceso de aprendizaje. Conclusión: La planificación y discusión de estrategias académicas en el proceso de enseñanza-aprendizaje están motivadas por acciones de educación continua para docentes.

Palabras clave: Enseñanza, Educación universitária, Profesores, Nutricionista.

\section{INTRODUÇÃO}

No Brasil, a formação profissional vem motivando mudanças no ensino superior, em especial à formação superior em saúde pelo foco humanista ao alcance da integralidade a atenção à saúde, características indispensáveis aos serviços do Sistema Único de Saúde (SUS) (SANTOS KT, et al., 2013).

A construção do conhecimento no processo participativo permite uma reflexão crítica da realidade. Desta forma deve haver precocidade na interação ativa entre o aluno, a população e os profissionais de saúde, no foco dos problemas reais e responsabilidades crescentes (PIMENTEL EC, et al., 2015; BALDOINO, 2016).

Desta forma, as Instituições de Ensino Superior (IES) diante de seus projetos político-pedagógicos vislumbram a reformulação curricular, estendendo a atenção a diversificação dos cenários de prática com uma das estratégias para aproximar os estudantes da vida cotidiana da população e desenvolver um olhar crítico, no cuidado dos reais problemas da sociedade (PIMENTEL EC, et al., 2015).

Neste contexto, cabe ressaltar o Estágio Curricular Supervisionado (ECS) como instrumento integrador, fomentando o incentivo à universidade ao repensar seu processo formador impactando no desenvolvimento científico e tecnológico.

Ao discente vislumbra-se a oportunidade de se autodescobrir como profissional, de vivenciar habilidades como responsabilidades que thes são conferidas e liderança de equipe, tão essenciais para a formação do futuro profissional. Assim, a instituição de ensino deve também ser norteadora na formação de profissionais éticos, competentes e seguros (EVANGELISTA DL e IVO OP, 2014; MARRAN L, et al., 2015).

No foco proposital a este estudo está o curso de Nutrição, com as Diretrizes Curriculares Nacionais (DCN's) aprovadas pelo Ministério da Saúde (MS) em 2001, no foco das competências e habilidades ao alcance das necessidades sociais da saúde, com ênfase no SUS.

Excelente estratégia para redirecionar a formação dos profissionais na constituição de um novo paradigma para a educação, impulsionando a efetivação dos princípios do SUS e das demandas e necessidades de saúde da população (BRASIL, 2001). 
Assim, o ensino superior requer mudanças de ensino aprendizagem, que fomentem o protagonismo estudantil, concedendo autonomia aos estudantes. Nesse contexto, as novas competências, habilidades, valores e conhecimentos exigidos pelo mercado de trabalho e sociedade trouxeram reflexões a respeito da formação e prática profissional existente.

Buscando-se assim, um novo perfil dos profissionais de saúde, em que a universidade tem a incumbência de superar a dicotomia teoria-prática e o ensino tradicional centrado no modelo biomédico, capacitando para além do mercado de trabalho e reproduzindo habilidades de lidar com as dimensões subjetivas, sociais e culturais dos usuários. (RIBEIRO MA, et al., 2016).

Mediante tais fatos, aponta-se algumas questões, as quais foram tomadas como norteadoras desta investigação: Como os docentes de Nutrição percebem o processo ensino aprendizagem dos graduandos nos cenários de prática? Quais as estratégias pedagógicas de ensino adotadas nos campos de estágio? Quais as principais facilidades e dificuldades encontradas durante o desenvolvimento deste processo? Como são realizadas as avaliações da aprendizagem dos alunos e que instrumentos são utilizados para estas avaliações nestes cenários?

Efetivamente, há uma grande preocupação com a qualidade da formação do profissional nutricionista, motivo pelo qual o presente estudo, visa contribuir com a concepção dos aspectos que envolvem o ensino aprendizagem desses profissionais.

Dessa maneira, o objetivo geral do estudo visou analisar as concepções dos docentes a cerca do processo ensino aprendizagem nos cenários de prática na formação do profissional nutricionista durante o estágio supervisionado em nutrição social e desta forma vislumbrou-se contribuir para a constituição de um novo conhecimento, provocando reflexões e estimulando a tomada de consciência, mediante a proposição de estratégias de ensino que potencializem o ensino aprendizagem.

\section{MÉTODOS}

O estudo exploratório com abordagem qualitativa, aprovada pelo Comitê de Ética em Pesquisa sob o parecer n‥ 2102273, foi desenvolvida na Faculdade de Nutrição de uma universidade pública do Estado do Pará, no período de Novembro de 2017 a Março de 2018, amparada no Projeto Pedagógico do Curso (PPC) de Nutrição, concebido com base na Resolução CNE/CES no5 (07/11/2001) que instituiu as Diretrizes Curriculares Nacionais do Curso de Graduação em Nutrição e tomando por base nesse cômputo a Resolução CNE/CES no 3 (02/07/2007), em consonância com o Plano de Desenvolvimento Institucional (PDI) e Resolução n 3.633 (18/02/2008).

O Curso de Graduação em Nutrição contava no período da pesquisa com 26 professores efetivos e 07 substitutos. Dentre os efetivos, 05 estavam afastados de suas atividades como docentes por estarem cursando doutorado e 03 estavam afastados por motivo de licença maternidade. Portanto, a amostra inicial foi composta por 25 professores, sendo finalizada com 21, devido às dificuldades encontradas por parte dos docentes que se recusaram a participar.

Adotou-se para inclusão no estudo, docentes das atividades curriculares e/ou que estivessem acompanhando estágios nas Unidades Básicas de Saúde (UBS). E como critérios de exclusão adotou-se os docentes com outra formação superior na área da saúde. Foi utilizado como instrumento para coleta de dados um questionário autoaplicável com perguntas fechadas e abertas, distribuído junto com o Termo de Consentimento Livre e Esclarecido (TCLE).

As perguntas norteadoras, que compuseram o questionário, foram organizadas pelas temáticas, ensino aprendizagem nos campos de estágios; estratégias de ensino aprendizagem utilizadas; cenários de prática, atividades desenvolvidas, dificuldades e facilidades encontradas nesse processo; instrumentos de avaliação para averiguação das habilidades e competências exigidas pelas DCN's aos egressos da graduação de nutrição. Para verificação da adequação do referido instrumento, foi realizado um teste piloto com a participação de dois docentes a fim de conduzir os ajustes necessários frente aos assuntos abordados. Estes não fizeram parte da análise do estudo. 
As informações qualitativas apreendidas nas entrevistas foram organizadas em um banco de dados, preparado, especificamente, para o futuro processo de análise de conteúdo processada pelo software ALCESTE, o qual viabiliza uma análise lexical de coexistências em enunciados simples de um texto, possibilitando, assim, a formação de classes de palavras que representam formas de discurso sobre o tema de interesse da pesquisa, na identificação e da dimensão do texto como um todo, mediante a identificação do número total e tipos de palavras, explorando a riqueza do vocabulário utilizado na produção de uma resposta ou um discurso. (CAMARGO VB, 2005; AZEVEDO DM, et al., 2013).

A organização dos dados foi viabilizada por meio de análises estatísticas e matemáticas, fornecendo o número de classe, as relações existentes entre as mesmas, as divisões realizadas no texto até a formação de classes, as formas radicais e palavras associadas com seus respectivos valores de qui-quadrado $\left(\mathrm{x}^{2}\right)$, além do contexto semântico de cada classe (FONSECA LM, 2013; AZEVEDO DM, et al., 2013).

O programa segmenta o material a ser analisado em grandes unidades denominadas Unidades de Contexto Inicial (UCl's), constituídas por entrevistas de diferentes sujeitos reunidas em um mesmo corpus, respostas específicas, normalmente abertas, de questionários e textos.

Por conseguinte, o texto é dividido em segmentos, denominados de Unidades de Contextos Elementares (UCE's) de algumas linhas, dimensionadas pelo programa em função do tamanho do corpus e correspondem ao material discursivo ou escrito referente à formação das classes (CAMARGO VB, 2005).

Deste modo, o número de classes resultante da análise, as formas reduzidas, o contexto semântico e as UCE's características de cada classe estabelecida pelo programa informático, são analisadas a fim de explicitar o conteúdo presente no mesmo, denominando e interpretando cada classe a partir de todas as informações fornecidas pelo software (FONSECA LM, 2013).

\section{RESULTADOS E DISCUSSÃO}

As verbalizações, analisadas pela metodologia do software ALCESTE revelou que o corpus, deu origem às $21 \mathrm{UCl}$ 's, alcançando 1.961 palavras diferentes, sendo descartadas as de frequência inferior a quatro. Assim, considerou 341 palavras alcançáveis dando sentido do objeto da comunicação, 120 instrumentais como articuladores discursivos fundamentais à organização do texto e 40 variáveis.

A análise hierárquica organizou o material textual em 265 UCE's com a divisão em cinco classes de segmentos de texto em partes distintas expondo as ideias e os temas mais dominantes no corpus textual, além disto, detalha a intersecção das classes.

As cinco classes geradas pela CHD abrangeram contextos semânticos específicos: Classe 1 (36\%): Importância do ECS no processo ensino aprendizagem; Classe 2 (14\%): Descrição dos cenários, dificuldades e facilidades dos locais de Estágio; Classe 3 (14\%): Estratégias de ensino para melhor interação com o discente; Classe 4 (14\%): Instrumentos utilizados nos processos de avaliação dos alunos; Classe 5 (22\%): Critérios avaliados nos discentes durante o estágio curricular supervisionado em nutrição social.

$\mathrm{Na}$ CHD o software também possibilitou a divisão final das classes representada graficamente por um Dendograma de leitura da esquerda para direita e a identificação lexical contida em cada uma das classes (Figura 1). 


\section{Revista Eletrônica Acervo Saúde / Electronic Journal Collection Health ｜ ISSN 2178-2091}

Figura 1 - Dendograma de Classificação Descendente das 265 Unidades de Contextos Elementares. ALCESTE. Belém - PA, 2020.

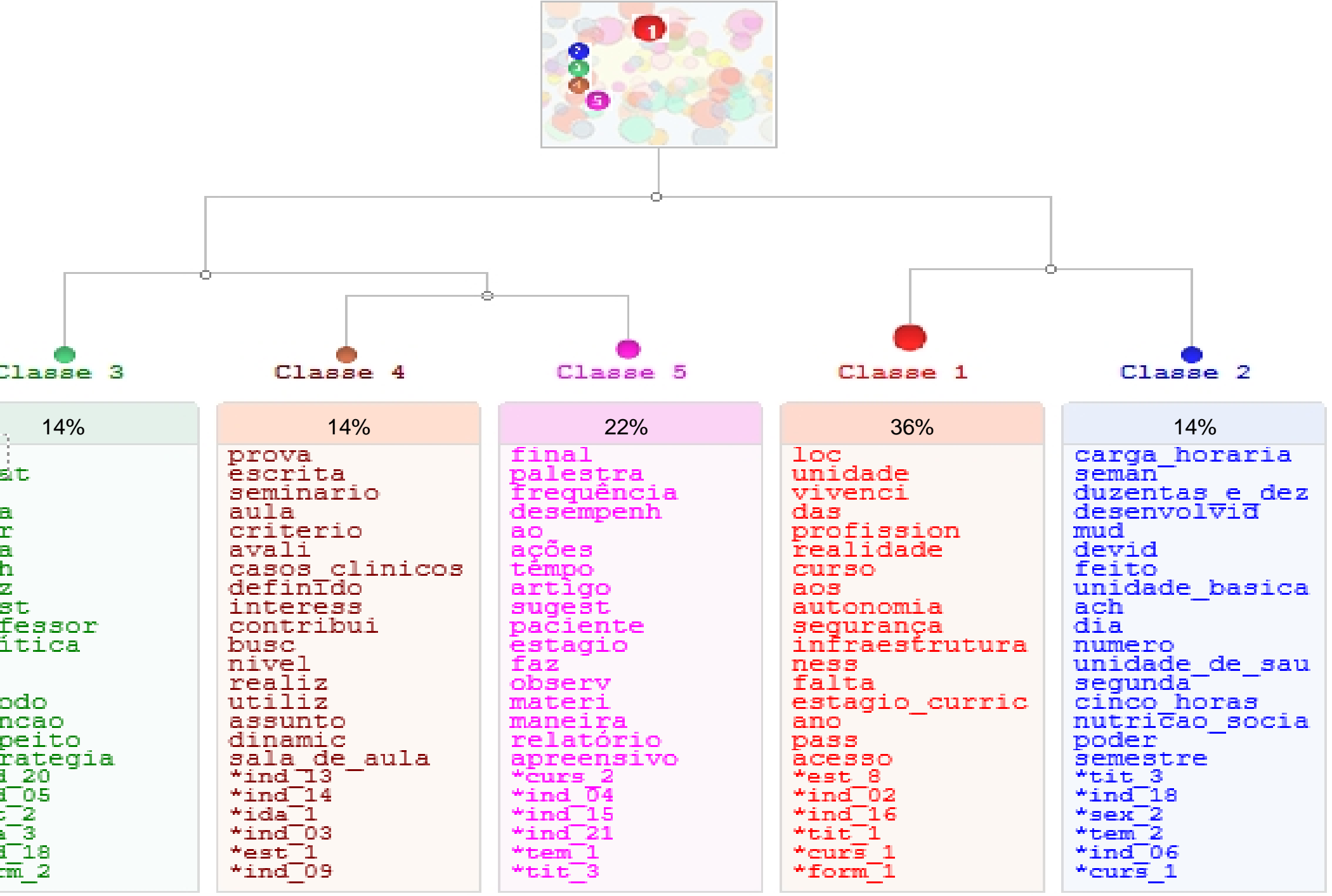

Fonte: MELO MF, et al., 2020 
Para cada classe detalhada abaixo, as dimensões exemplificadas nas UCE's foram contempladas com base nos registros dos conteúdos coletados e transcritos ao software ALCESTE.

A Classe 1, mais específica sobre a importância do ECS no processo ensino aprendizagem, é a primeira a ser destacada na árvore de classificação hierárquica, portanto, seu vocabulário é mais homogêneo, sendo representada por 64 UCE's, classificadas ou $36 \%$, e nela foram selecionadas 73 palavras com destaque para as que tiveram maior frequência ou qui-quadrado. As mais significativas desta classe, são termos reduzidos como: loc, unidade, vivenci, das profission, realidade. Verificou-se que o termo loc, forma reduzida das palavras locais e local, apresentou maior valor de $X^{2}=25$, indicando que este termo possui maior associação com a Classe 1.

As expressões enalteceram que o ECS se firma como uma atividade de consolidação do conhecimento adquirido no curso da formação acadêmica, atuando como um mediador da integração ensino-serviço e, como um importante componente curricular de prática imprescindível, pois prepara os estudantes para o trabalho e à realidade social, visando ao aprendizado de competências próprias da atividade profissional e à contextualização curricular.

Considera-se, portanto, o ECS como um ato educativo, elemento constituinte do PPC, com foco no aperfeiçoamento técnico e a compreensão da realidade social, cultural, econômica e epidemiológica, para a formação acadêmica baseada nas necessidades da sociedade (BRASIL, 2010, MARRAN L, et al., 2015). As DCN's, recomendam a formação na diversidade de cenários, assim a inserção de discentes em campos de práticas integram o compartilhamento de conhecimentos com docentes, preceptores, profissionais dos serviços e comunidade. (CARVALHO SB, et al., 2015).

Nessa perspectiva, ressalta-se que o processo de ensino aprendizagem que pode ser estabelecido a partir da inserção dos alunos nestes cenários de prática, tende a favorecer novas formas de organização do trabalho, em busca de uma melhor qualificação para o profissional (BALDOINO, 2016).

A Classe 2, composta por 26 unidades, ou 14\% das UCE's classificadas, com 65 palavras, gerou os mais significativos termos como: carga horária, semana, duzentas e dez horas, o, desenvolvido, mudado. Verificou-se que o termo, carga horária, apresentou maior valor de $X^{2}=36$. Esta classe trata das verbalizações sobre a descrição dos cenários, dificuldades e facilidades dos locais de ECS em Nutrição Social.

A partir dos registros, identificou-se que a UBS é o principal cenário de prática para a realização das atividades inerentes ao ECS em Nutrição Social, mostrando consonância com o que é preconizado pelo SUS, no nível primário de atenção à saúde, para o alcance das habilidades e competências, como: conhecer as atividades desenvolvidas por um nutricionista e atender usuários de todos os ciclos da vida, em uma UBS.

A ausência de estruturação adequada prejudica a articulação das instituições de ensino com os serviços de saúde, o que implica negativamente no processo ensino aprendizagem (MARRAN L, et al., 2015). A sensibilização e acolhimento da equipe multidisciplinar juntamente com a comunidade são imprescindíveis para que as atividades práticas nos cenários tenham um bom desenvolvimento, pois interfere diretamente na desenvoltura e participação mais ativa dos discentes, proporcionado ainda uma efetiva aprendizagem das atividades articuladas durante o estágio (WERNECK MAF, et al., 2010).

A Classe 3, a qual trata das verbalizações sobre as estratégias de ensino para melhor interação com o discente, deteve 24 unidades, ou $14 \%$ das UCE's classificadas, e nela foram selecionadas 49 palavras com destaque para as mais significativas: fala, debate, eu, vai, área, quer. Verifica-se que o termo fala apresentou maior valor de $\mathrm{X}^{2}=26$.

Percebeu-se que os docentes, buscam por meio da utilização de estratégias de ensino não convencionais uma interação com os alunos, a fim de obter uma atuação mais participativa, questionadora, interativa e uma maior assimilação do conteúdo. Portanto, é necessário desenvolver estratégias a partir da realidade do estudante, despertando sua curiosidade pelo ensino, instigando-o a participar da construção da própria aprendizagem e tornando-o autor de sua formação. 
Assim ressalta-se o uso das rodas de conversas, para expressar opiniões, compartilhar conhecimentos prévios, conceitos e concepções sobre algum tema proposto, permitindo também um melhor conhecimento ao docente a respeito do perfil da turma, contribuindo para escolhas de outras estratégias pedagógicas no processo ensino aprendizagem.

Para que no processo ensino aprendizagem ocorra interação e comprometimento, os docentes devem estar capacitados para despertar o interesse nos discentes, para isso o auxílio de novas metodologias de ensino e sua inserção no processo de trabalho das equipes passa a ser ferramenta importante, para que os discentes possam construir novos conhecimentos e serem agentes transformadores da realidade. (LUZ MMA, et al., 2015).

Contudo, não existe uma pedagogia perfeita para ser desenvolvida em todos os momentos e para todos os conteúdos. A estratégia a ser utilizada vai depender dos objetivos estabelecidos pelo professor e das habilidades a serem desenvolvidas em cada componente curricular na articulação do processo de ensino para estimular o aprendizado. O professor precisa ser capaz e possuir a habilidade em identificar e escolher o método mais adequado para cada situação durante o processo de educar (MAZZIONI S, 2013).

Neste ínterim, com as exigências de um novo perfil profissional, o docente deve ter a preocupação em propiciar, ao estudante, condições de refletir acerca de: sua postura, responsabilidades, mudanças e possibilidades, desenvolvendo sua capacidade de pensamento, reflexão, análise e síntese (CAVALCANTE LIP, et al., 2011; ALMEIDA, 2012).

$\mathrm{Na}$ Classe 4, a qual trata das verbalizações dos sujeitos da pesquisa sobre os instrumentos utilizados nos processos de avaliação dos alunos do curso de Nutrição, deteve-se 25 unidades, ou 14\% das UCE's classificadas, e foram selecionadas 56 palavras, como prova, escrita, seminário, aula, critério, avaliação, com destaque para prova com a maior frequência ou $X^{2}=57$.

A avaliação do desempenho do aluno é parte do processo ensino aprendizagem e para atingir sua finalidade educativa, deve ser coerente com os princípios pedagógicos e sociais do projeto de formação adotado.

A avaliação oportuniza ao professor subsídios que levam a uma reflexão continuada sobre seu papel docente, em relação à escolha de competências, objetivos, conteúdos e formas de ensinar e avaliar, dando suporte de quais aspectos deve ser revisado, adaptados ou identificados como os mais apropriados para 0 processo que consiga atingir uma compreensão individual e coletiva da turma. (DARIDO SC e RANGEL IC, 2014). Alguns educadores tendem a levar seus esforços ao aspecto quantitativo, desvalorizando o aspecto qualitativo, via de diagnóstico da evolução dos alunos. Isso pode ocorrer pois a avaliação qualitativa requisita mais raciocínio e tempo de elaboração (MORETTO VP, 2005; BARBARA MRLS e MARTINS APR, 2012).

A Classe 5 deteve 35 unidades, ou 22\% das UCE's classificadas, e foram selecionadas 61 palavras com destaque para final, palestra, frequência, desempenho, ao, ações. O termo final foi o de maior frequência com $X^{2}=28$. Esta classe trata das verbalizações sobre os critérios avaliados nos discentes durante 0 estágio curricular supervisionado em nutrição social, complementando a discussão iniciada na Classe 4.

Constatou-se que os docentes fazem avaliação dos seus alunos em campo de estágio, de forma processual, procedida diariamente, avaliando a atitude do aluno e a habilidade no desenvolvimento das atividades propostas.

Os docentes mencionaram que o desempenho do discente é avaliado por meio utilização de métodos integrados, formativos, da combinação de conhecimentos dos diferentes conteúdos, sua compreensão, resolução de problemas, habilidades técnicas, e atitudes. Constatando que a avaliação não deve estar restrita a um método, técnica ou instrumento, e sim centrada na pluralidade de procedimentos de verificação da aprendizagem. Neste sentido é importante a realização de avaliações diagnósticas, formativas, com os necessários feedbacks, e avaliações somativas, na busca do rendimento, facilidade de compreensão, nível de conhecimentos teóricos, organização, método, iniciativa, independência e ética. Além de atitudes comportamentais como assiduidade, pontualidade, cooperação e responsabilidade. 
A avaliação da aprendizagem configura um componente intrínseco e vital do ensino aprendizagem, sendo responsabilidade do docente em seus diversos ambientes de trabalho, podendo ser considerada uma das tarefas mais complexas da docência, e que implica diretamente o sucesso acadêmico dos alunos (LUCKESI CC, 2011).

Os discentes durante suas atividades no estágio passam por avaliação formativa, o que permite constatar se, de fato, estão alcançando os objetivos e resultados propostos durante o desenvolvimento das atividades planejadas. Desta forma, cabe destacar que esta avaliação é norteadora tanto do estudo do aluno quanto das atividades desempenhadas pelos docentes, uma vez que permite que o docente detecte e identifique deficiências na forma de ensinar, possibilitando reformulações no seu trabalho didático, visando a aperfeiçoá-lo (ARAÚJO MML, 2014).

Esta pesquisa permitiu uma melhor compreensão dos diversos aspectos que envolvem a dinâmica do ensino aprendizagem desenvolvido no curso de graduação em Nutrição em uma universidade pública do Estado do Pará, na percepção dos docentes.

Os resultados da pesquisa também apontam a necessidade de adoção de uma formação permanente do docente para atuação na prática, rompendo assim a dicotomia entre teoria e prática, desarticulando a ordem dos conteúdos teóricos e práticos ministrados numa sequência que dificulta tornar 0 aprendizado significativo, bem como realização de planejamento conjunto das ações com os serviços de saúde utilizados como cenários de prática, sensibilizando-os a se fazerem parte integrante desse processo ensino aprendizagem.

\section{CONCLUSÃO}

O docente deve constantemente participar de oportunidades que contribuam para seu crescimento profissional no que se refere à área pedagógica, reforçando a ideia de que a formação pedagógica enriquece e muda o pensamento do educador em relação ao processo de ensino aprendizagem. $\mathrm{E}$ que a instituição de ensino utilize estratégias de incentivo que aumente a participação docente nas ações de formação continuada, além de reforçar a importância do planejamento e discussão das atividades acadêmicas a serem executadas.

\section{AGRADECIMENTOS}

Ao Programa de Pós-Graduação Ensino em Saúde na Amazônia (PPG-ESA) da Universidade do Estado do Pará (UEPA).

\section{REFERÊNCIAS}

1. ALMEIDA MM. A escassez de líderes no mercado de trabalho: o papel do professor universitário na formação deste profissional pode colaborar para a mudança do cenário atual. Gestao Soc. 2012; 1(1): 1-9.

2. ARAÚJO MML. Análise do ensino aprendizagem nos estágios de graduação em enfermagem: percepção dos docentes. (Mestrado Profissional Ensino na Saúde). Universidade Estadual do Ceará. Fortaleza. CE. 2014. 114.

3. AZEVEDO DM, et al. Uso do Alceste na análise de dados qualitativos: contribuições na pesquisa em enfermagem. Revista de Enfermagem de UFP, 2013, 7: 5015-22.

4. BALDOINO ASL. A integração ensino-serviço no processo de formação superior em saúde na Universidade Federal da Bahia. (Programa de Pós-graduação Estudos Interdisciplinares). Universidade Federal da Bahia. Salvador. BA. 2016; 81.

5. BARBARA MRLS, MARTINS APR. Avaliação: uma prática constante no processo de ensino e aprendizagem. Faculdade Católica de Uberlândia. Revista da Católica, 2012, 3: 1-12.

6. BRASIL, Ministério da Educação. Conselho Nacional de Educação. Câmara de Educação Superior. Resolução CNE/CES no 5, de 07 de novembro de 2001. Institui Diretrizes Curriculares Nacionais do Curso de Graduação em Nutrição. Diário Oficial da União: 1:39.

7. BRASIL. Ministério do Trabalho e Emprego. Nova cartilha esclarecedora sobre a lei do estágio. Lei 11.788 , de 25 de setembro de 2008. Brasília, 2010; 32.

8. CARVALHO, SOB, et al. Parceria ensino e serviço em unidade básica de saúde como cenário de ensinoaprendizagem. Trabalho, Educação e Saúde. Rio de Janeiro, 2015; 13(1): 123-144. 
9. CAMARGO VB. ALCESTE: um programa informático de análise quantitativa de dados textuais. In: MOREIRA ASP. (Org.). Perspectivas teórico-metodológicas em representações sociais. João Pessoa: UFPB/Editora Universitária; 2005: 511-39.

10. CAVALCANTE, L.I.P., et al. A docência no ensino superior na área de saúde: formação continuada e desenvolvimento profissional em foco. Rev Eletr Pesquieduca, 2011; 3(6):162-82.

11. DARIDO SC, RANGEL IC. Educação Física no ensino superior: educação física na escola, implicações para prática pedagógica. 2. ed. Rio de Janeiro: Guanabara Koogan, 2014: 21-33.

12. EVANGELISTA DL, IVO OP. Contribuições do estágio supervisionado para a formação do profissional de enfermagem. Revista Enfermagem Contemporânea, 2014; 3(2): 123-130.

13. FONSECA LM. Termos e expressões utilizados por familiares ao relatarem suas experiências nos diferentes momentos do adoecimento mental. (Mestrado em Enfermagem Psiquiátrica). Escola de Enfermagem, Ribeirão Preto, São Paulo, 2013; 77.

14. LUCKESI CC. Avaliação da Aprendizagem. Componente do Ato Pedagógico. São Paulo: Cortez, $2011 ; 448$.

15. LUZ MMA, et al. A formação do profissional nutricionista na percepção do docente. Interface - comunicação, saúde, educação, Botucatu, 2015; 19(4): 589-601.

16. MARRAN L, et al. As políticas educacionais e o estágio curricular supervisionado no curso de graduação em enfermagem. Trabalho, Educação e Saúde. 2015; 13(1): 89-108.

17. MAZZIONI S. As estratégias utilizadas no processo de ensino-aprendizagem: concepções de alunos e professores de ciências contábeis. Rev Eletron Adm Tur, 2013; 2(1): 93-109.

18. MORETTO VP. Prova, um momento privilegiado de estudos e não um acerto de contas. Rio de Janeiro: Lamparina Editora, 2005; 192.

19. PIMENTEL EC, et al. Teaching and Learning in Supervised Internship: Integrated Internship in Health. Rev. bras. educ. med. $2015 ; 39(3)$ : 352-358.

20. PROJETO PEDAGÓGICO DO CURSO DE NUTRIÇÃO. UNIVERSIDADE FEDERAL DO PARÁ. 2010. 57 p.

21. RIBEIRO MA, et al. Vivências e estágios na realidade do sistema único de saúde (VER-SUS) como agente promotor de mudanças na formação de graduação e nas práticas profissionais. Saúde e Transformação Social. 2016; 7(1): 109-119.

22. SANTOS KT, et al. Percepção discente sobre a influência de estágio extramuros na formação acadêmica odontológica. Rev. odontol. UNESP, 2013; 42, n. 6: 420-425.

23. WERNECK MAF, et al. Nem tudo é estágio: contribuições para o debate. Ciência da Saúde Coletiva. 2010. 15(1): 221-31.

24. ZEFERINO AMB, PASSERI SMRC. Avaliação da aprendizagem do estudante, Cadernos ABEM, 2007; 3, Rio de Janeiro/RJ: 39-43. 